\title{
LA RADIOGRAFÍA CEFÁLICA: MÁS ALLÁ DE UNA MEDIDA CEFALOMÉTRICA
}

\author{
Ana María Quintero, Odon. ${ }_{1}$, Bibiana Escobar, Odon. ${ }_{1}$, Natalia Vélez Trujillo*, Odon. Ortod. ${ }_{1}$
}

${ }_{1}$ Universidad Cooperativa de Colombia, sede Medellín, Colombia

Recibido: 26 de noviembre del 2012 Aprobado: 24 de enero del 2013

* Autor de contacto: Natalia Vélez, Facultad de Odontología, Universidad Cooperativa de Colombia, 2706466, Carrera 47 n. ${ }^{\circ} 37$ sur 18 , Medellín, Colombia, correo electrónico: natalia.velez@campusucc.edu.co

Cómo citar este artículo: Quintero AM, Escobar B, Vélez Trujillo N. La radiografía cefálica: más allá de una medida cefalométrica. Rev. Nac. Odontol. 2013 diciembre; 9 (edición especial): 7-15.

Resumen. La práctica ortodóncica reconoce la importancia de la radiografía cefálica lateral, aunque en realidad la limita a un análisis cuantitativo, por lo cual se realizó una revisión de literatura con el fin de valorar las características morfológicas que a simple vista pueden ser evaluadas en la radiografía y que son herramientas para predecir factores de riesgo, pronóstico y estabilidad. Se concluye que la radiografía cefálica lateral da una visión más amplia de los patrones de crecimiento craneofacial, los cuales pueden ser modificados por diversas estructuras - como la base del cráneo, la postura craneocervical, las vías aéreas y la posición del hueso hioides, sin dejar de valorar las vértebras cervicales y la morfología de la sínfisis mandibular- que se constituyen en factores diagnósticos importantes en el momento de tomar decisiones clínicas. Por tanto, la radiografía cefálica lateral brinda información cualitativa importante que no se puede obviar y que permite llegar a un diagnóstico más acertado.

Palabras clave: base del cráneo, hueso hioides, postura craneocervical, radiografía cefálica, sínfisis mandibular, vértebras cervicales, vías aéreas.

\section{The Cephalic Radiograph: Beyond a Cephalometric Measure}

Abstract. Orthodontic practice recognizes the importance of the cephalic lateral radiograph, although it is actually limited to quantitative analysis. A literature review was made to assess the morphological characteristics that, at first glance, can be evaluated in the radiography and constitute tools for predicting risk factors, prognosis, and stability. The conclusion is that the cephalic lateral radiograph gives a broader view of craniofacial growth patterns, which can be modified by various structures such as skull base, craniocervical posture, airways, and position of the hyoid bone; this without losing sight of the cervical vertebrae and the morphology of the mandibular symphysis, which are important diagnostic factors when making clinical decisions. Cephalic lateral radiography therefore provides important qualitative information that cannot be ignored and which enables accurate diagnosis.

Keywords: skull base, hyoid bone, cranio-cervical posture, cephalic radiography, mandibular symphysis, cervical vertebrae, airways. 


\section{Introducción}

La práctica ortodóncica reconoce la importancia de la radiografía cefálica lateral, aunque en realidad la limita a un análisis, que para ser satisfactorio depende de la fiabilidad y la interpretación de las medidas cefalométricas.

Con el advenimiento de los análisis cefalométricos, el especialista en ortodoncia es quien hace útil y válida esta ayuda en la difícil tarea diagnóstica, apoyándose tanto en su conocimiento como en la interpretación de las medidas cuantitativas, además de evaluar los factores de riesgo que puedan afectar el pronóstico y las estrategias terapéuticas. La valoración radiográfica se basa en dos supuestos: primero, que el patrón de crecimiento facial puede ser estimado, y segundo, que es probable que este patrón continúe [1].

El presente artículo incluye una revisión bibliográfica para determinar las características morfológicas craneofaciales que a simple vista pueden ser evaluadas en una radiografía cefálica y que son herramientas para predecir factores de riesgo, pronóstico y estabilidad. Estas son: base del cráneo, postura craneocervical, vértebras cervicales, posición del hueso hioides, vías aéreas y morfología de la sínfisis mandibular, que van más allá de una simple medida cefalométrica y que no siempre son evaluadas.

\section{Base del cráneo}

Debido a su ubicación entre el cráneo, el tercio medio facial y la fosa glenoidea, la base craneal tiene el potencial de influir en el crecimiento del cráneo y la cara $[2,3]$, razón por la cual se considera que influye en el patrón esquelético y en el tipo de maloclusión $[4,5]$. Frente a las funciones neuromusculares alteradas o los tratamientos de ortodoncia interceptiva o correctiva, esta es la estructura craneofacial más estable $[3,6]$.

Para los ortodoncistas, biólogos y antropólogos, los patrones de normalidad en el desarrollo de la base del cráneo deben ser conocidos para comparar las variaciones en el patrón de crecimiento en los pacientes mediante la superposición de radiografías sucesivas $[3,6]$.

El crecimiento de la base del cráneo está relacionado con el desarrollo general del cuerpo, que se establece por medio de un equilibrio complejo que incluye la ampliación de los senos frontales, la remodelación de la superficie en la región del nasion y el crecimiento intersticial en la sincondrosis esfeno-occipital $[2,7,8]$.

Durante la vida intrauterina se alcanza el 56\% de la longitud adulta de la base del cráneo. Ohtsuki y colaboradores concluyen que la tasa más rápida de crecimiento de esta se da entre el nacimiento y los 2 años de edad [2]. Hoyte reporta que la mayor parte de crecimiento sagital de la base craneal anterior se completa a los 7 años de edad, tiempo en que el primer molar permanente ya ha erupcionado $[2,6,9]$.

El desarrollo de la región intermedia aumenta en longitud debido al crecimiento de la sincondrosis esfeno-occipital, que sólo sirve para alargar el clivus y la parte basilar del hueso occipital, el cual es desplazado dorsalmente como consecuencia del incremento de la distancia entre silla y basion $[4,6,9,10]$. Este crecimiento es mayor en hombres que en mujeres en cuanto a tiempo y cantidad [3].

La relación entre la base y la bóveda craneal genera una rotación de las estructuras faciales $[2,11]$ dando cambios angulares que se producen entre la base craneal anterior y posterior, y están definidos por los planos $\mathrm{Na}-$ sion-Silla-Basion (N-S-Ba) y Nasion-Silla-Articular (N-S-Ar) (figura 1) [4, 12, 13]. Al nacer, el ángulo N-SBa es de aproximadamente $142^{\circ}$, a los 5 años es de $130^{\circ}, y$ de los 5 a los 15 años es relativamente estable [4, 14]. Kerr y Hirst reportaron que a los 5 años de edad el ángulo de la base craneal es un indicador del tipo de oclusión que tendrán los pacientes a los 15 años de edad en un 73\% [14].

Un ángulo de la base del cráneo inferior a $120^{\circ}$ se considera cerrado; este guarda relación con un perfil cóncavo, una clase III esquelética y un desplazamiento anterior del cóndilo. Por el contrario, un ángulo de base de cráneo mayor a $125^{\circ}$ se considera abierto y produce una ubicación posterior y superior de la cavidad glenoidea y, por consiguiente, de la articulación temporomandibular (ATM), ubicando la mandíbula en una posición retrusiva y un patrón clase II esquelético $[4,14,15]$.

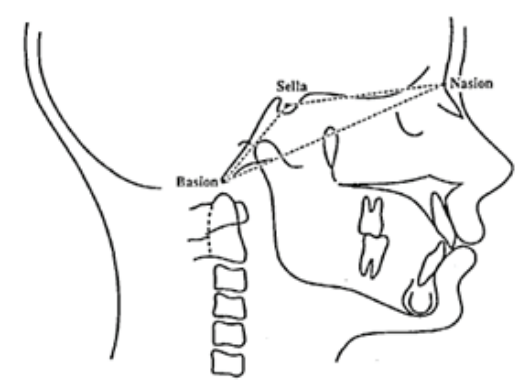

Figura 1. Puntos cefalométricos del ángulo de la base craneal Fuente: Henneberke y PrahI-Andersen, 1994 [3]

Se concluye que la deflexión de la base del cráneo no es un determinante ni puede ser considerada como factor etiológico único de los patrones de crecimiento de 
las diferentes estructuras del complejo craneofacial ni del tipo de maloclusión [11], ya que su crecimiento es similar en individuos clase I y clase II esqueléticos, y la correlación entre la longitud como tal de la mandíbula y la base de cráneo es poca en los pacientes clase III, aunque se asocian con un tamaño disminuido de la base del cráneo posterior $[2,11,13]$; por tanto, es fundamental hacer una valoración individual del paciente, ya que en la literatura no hay una correlación directa y concluyente [11].

\section{Postura craneocervical}

La articulación temporomandibular, la articulación del eje occipital-atlas y el hueso hioides, con su sistema suspensor unido con la columna vertebral por músculos y ligamentos, constituyen el sistema cráneo cervico mandibular, lo que contribuye a que la postura cervical pueda influir en la morfología craneofacial y la función respiratoria [16].

La postura corporal equilibrada consiste en la alineación del cuerpo con una máxima eficiencia tanto fisiológica como biomecánica, lo que reduce el estrés y las sobrecargas por los efectos de la gravedad. En la postura correcta, la línea de gravedad pasa a través de los ejes de todas las articulaciones con los segmentos corporales alineados verticalmente y la columna cervical formando una lordosis (leve curvatura de convexidad anterior). Esta postura está dada por los músculos de la región anterior, que mantienen erectos la cabeza y el cuerpo, mientras que los de la región posterior - los cervicales y occipitales - contrabalancean las fuerzas de gravedad en todo el cuerpo. El equilibrio de la parte más baja del cráneo depende de los músculos masticatorios y los supra e infrahioideos [17].

La postura cráneo cervical es el último eslabón de la postura de la columna vertebral, es decir, el eje del cuerpo. Para evaluarla, la radiografía se debe tomar con una posición natural de la cabeza, la cual se logra de forma reproducible con el paciente de pie; esta se encuentra regulada por dos mecanismos: primero, las entradas nerviosas, y segundo, la entrada desde el sistema de enderezamiento visual, cuando el paciente fija la mirada y hace un ajuste postural fino del sistema [18].

Una alteración postural común, es el posicionamiento anterior de la cabeza, que se caracteriza por una extensión dorsal de la columna cervical superior (C1C3), acompañada por una flexión de la columna cervical inferior (C4-C7), lo que genera un aumento de la curvatura cervical, denominada hiperlordosis donde existe hiperactividad de los músculos esternocleidomastoideo y trapecio superior, generando una disfunción en la cabeza y el cuello, retrusión mandibular, un desarrollo facial vertical, un posicionamiento posterior de la ATM, un ángulo goníaco aumentado y una posible obstrucción de vías aéreas superiores; en otras palabras, un patrón esquelético de clase II $[16,17]$.

Los cambios en la angulación y la inclinación de la posición natural de la cabeza y la columna cervical se han asociado con el grado y la dirección de la rotación de la mandíbula en crecimiento [19]. Cuando el ángulo cráneocervical es evaluado de 2 a 4 años antes del pico de crecimiento puberal, puede proporcionar información predictiva del desarrollo facial posterior [18].

Entre los factores desencadenantes en los cambios de la postura de la cabeza, se encuentra la obstrucción de las vías aéreas superiores y la respiración oral, debido a que afectan tanto la postura de la lengua y de la mandíbula, como el equilibrio de los músculos orales y periorales $[1,18,20]$.

El ángulo cráneocervical está dado por la relación de la postura de la cabeza a una línea que representa la columna cervical, y el cual se forma por la línea de la base anterior del cráneo con la tangente posterior de la apófisis odontoides (sNSL/OPT) (figura 2). Un ángulo pequeño y una columna cervical superior inclinada hacia atrás, se asocian con el desarrollo facial horizontal - caracterizado por reducir el desplazamiento de la ATM, un aumento del crecimiento en longitud del maxilar superior y una rotación hacia adelante de la mandíbula-, mientras que un ángulo cráneocervical grande y una posición vertical de la columna cervical superior, se asocian con el desarrollo vertical facial que se caracteriza por el desplazamiento hacia atrás de la ATM, la reducción de crecimiento en la longitud del maxilar superior y la rotación de la mandíbula $[1,18]$.

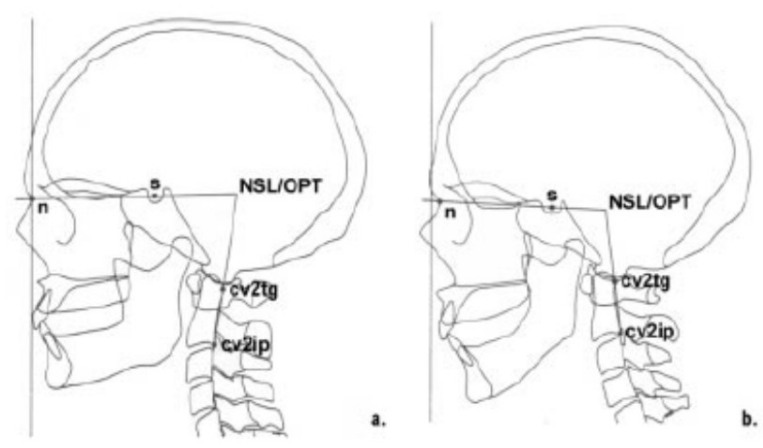

Figura 2. Ángulo cráneocervical. a. Ángulo cerrado. b. Ángulo abierto Fuente: Solow y Sandham, 2002 [8] 
Houston sugirió que el crecimiento de la columna cervical es el factor principal que determina la altura facial anterior. Después de la pubertad, el crecimiento vertical de la cara tiene una alta correlación con el crecimiento del cuello, de modo que los pacientes leptoprosopos a menudo tienen una columna cervical recta y larga, mientras que en los braquicefálicos parece ser curva [21].

El equilibrio biomecánico de la cabeza se consigue por medio de un sistema de palancas del tipo interfijo, en el cual la articulación atlanto-occipital es el punto de apoyo [17]. La evaluación de ciertas características morfológicas de las vértebras cervicales tiene valor en el pronóstico en cuanto al patrón esquelético sagital [22]. La morfología del atlas, primera vértebra cervical, guarda correlación con la postura cráneocervical, particularmente con respecto a la dimensión vertical del arco dorsal del atlas. Von Treuenfels observó que la inclinación del atlas está asociada con la posición sagital de la mandíbula, en la que su arco ventral alcanza una posición más craneal en los pacientes prognáticos. Kylamarkula y Huggare reportaron que la amplitud vertical del arco dorsal de este, era significativamente más bajo en pacientes con adenoides hipertróficas y mandíbula retrognática y más común en mujeres con mordida abierta $[21,22]$.

La condición oclusal de los pacientes tiene una etiología multifactorial, y la postura cráneocervical es uno de los factores que podrían influir en la aparición de apiñamiento en el arco inferior. Una posición extendida de la cabeza de forma prolongada altera el equilibrio entre los labios, las mejillas, la lengua y los incisivos inferiores, patrón que se establece en los pacientes con obstrucción de vías aéreas superiores y respiración oral [16].

\section{Vertebras cervicales}

Para evaluar la madurez esquelética se han utilizado una variedad de indicadores biológicos, como el aumento de la altura corporal; la maduración de los huesos de mano y muñeca; el desarrollo dental y su erupción; la menarca; los cambios en la voz y la maduración de las vértebras cervicales $[23,24]$.

El método de maduración vertebral cervical (CVM) ha demostrado ser eficaz para evaluar el pico de crecimiento del cuerpo mandibular en pacientes en crecimiento $[25,26]$. La clasificación de los estadios de osificación se basa en los cambios de maduración tanto en el tamaño como en la forma de las vértebras cervicales C2 a la C6 [26, 27].

Los estadios de osificación de las vértebras cervicales (CVM) descrito por Lamparski [28] son los siguientes:

1. Iniciación (CS1): los bordes inferiores de la C2, C3 y C4 están planas. La C3 y C4 tiene forma trapezoidal y sus bordes superiores están afilados desde posterior hasta anterior. Del 80 al 100\% del crecimiento puberal no se ha presentado.

2. Aceleración (CS2): una concavidad está presente en el borde inferior de C2. Los cuerpos de C3 y C4 son todavía de forma trapezoidal. El 65-85\% del crecimiento puberal está por darse.

3. Transición (CS3): hay concavidades en los bordes inferiores de C2 y C3. Los cuerpos vertebrales de C3 y C4 son rectangulares. Resta un 25-65\% de crecimiento puberal.

4. Desaceleración (CS4): se ven concavidades en los bordes inferiores de C2, C3 y C4. Los cuerpos vertebrales de C3 y C4 son más o menos cuadrados. Sólo queda un 10-25\% del crecimiento puberal por expresarse.

5. Maduración (CS5): se observan concavidades acentuadas en los bordes inferiores de C2, C3, C4, C5 y C6. Los cuerpos vertebrales de C3 y C4 son casi de forma cuadrada. En este estadio se presenta la maduración final con un 5-10\% restante de crecimiento puberal.

6. Completación (CS6): se ven concavidades profundas en los bordes inferiores de C2 a C6. Los cuerpos vertebrales son mayores verticalmente que horizontalmente. El crecimiento puberal ha terminado [26].

El mayor incremento en las variables cefalométricas de la longitud mandibular — tanto de la rama como del cuerpo- y la mayor aposición ósea en el cóndilo, tiene lugar en el intervalo de crecimiento de CS3 a CS4 en comparación con otros estadios [23, 24, 29].

La eficacia de los aparatos funcionales depende en gran medida de la capacidad de respuesta del cartílago del cóndilo y de la tasa de crecimiento de la mandíbula, que no es constante durante todo el desarrollo. Por tanto, evaluar el potencial de crecimiento individual y determinar el momento óptimo para iniciar un tratamiento, proporciona información esencial para la previsión de los resultados [24-27]. Por ejemplo, la corrección de la clase II esquelética, o las deficiencias en la altura de la rama mandibular, debe llevarse a cabo cuando la probabilidad de respuesta de crecimiento 
es alta, es decir, durante el periodo de crecimiento circumpuberal CS3 [25-30].

Los estudios han demostrado que el tratamiento de la maloclusión clase III esquelética es más efectivo en las etapas tempranas que en la dentición mixta tardía, lo mismo que en las deficiencias maxilares transversales. Se recomienda expandir la sutura media palatina antes del pico de crecimiento para lograr un efecto ortopédico, o más dentoalveolar, si se realiza durante o después de este [25,30]. El tratamiento prepuberal es efectivo tanto en el maxilar como en la mandíbula, aunque en el estadio puberal es efectivo sólo en la mandíbula [25].

Debido a que existen factores que afectan la precisión y reproducibilidad del método CVM — como son las mediciones subjetivas del clínico, las formas de las vértebras cervicales que tienen marcadas variaciones de un sujeto a otro, y la calidad de la radiografía-, es preferible correlacionarlo con otros indicadores de crecimiento en la evaluación de la maduración del esqueleto antes de tomar decisiones [31].

\section{Posición del hueso hioides}

El hioides es el único hueso del cuerpo que no tiene articulaciones óseas, y como es móvil puede sufrir constantes variaciones como respuesta fisiológica a los requerimientos funcionales de la deglución, la respiración y la fonoarticulación [32]. Dentro de sus funciones está prevenir la regurgitación, mantener la vía aérea - al disminuir la succión interna de las partes blandas- y mantener la posición postural vertical de la cabeza, relacionada con la curvatura de la lordosis cervical debido a su conexión a la faringe, la mandíbula y el cráneo, a través de músculos, ligamentos y la fascia cervical $[33,34]$.

Existen dos grupos musculares que realizan funciones concomitantes con el hueso hioides: los suprahioideos y los infrahioideos.

Los suprahioideos (milohioideo, genihioideo, digástrico y estilohioideo), tienen acción directa en el control de la dinámica mandibular, y los infrahioideos (esternohioideo, omohioideo, tirohioideo) establecen a su vez la función estabilizadora del hioides [32].

Durante el curso de la vida la posición normal del hueso hioides tiene un rango de variabilidad, por ser un fenómeno fisiológico relacionado con otros cambios anatómicos en la cara. Bench indicó que el hueso hioides desciende gradualmente desde una posición opuesta a la mitad inferior de $\mathrm{C} 3$ y la mitad superior de $\mathrm{C} 4$ a la edad de 3 años, y al frente de C4 en la edad adulta. El cuerpo está en relación con el ángulo anteroinferior de $\mathrm{C} 3$, mientras que las astas posteriores tienden a permanecer constantes entre la C2 y C3 (figura 3). Tsai reportó que estos cambios son mayores en las estructuras faríngeas de los hombres [33], y que estos movimientos del hioides no están correlacionados con la obesidad [35].

La inclinación del hioides varía con relación al patrón facial al seguir de cerca la inclinación axial de la mandíbula. Los pacientes con caras cortas tienen una posición del hueso hioides más cercana al plano mandibular y más posterior, es decir, hacia las vértebras cervicales. En contraste, los patrones faciales largos y los normales tienen una posición del hioides más inferior $\mathrm{y}$ anterior $[35,36]$.

$\mathrm{Al}$ evaluar la interacción entre la lengua, la posición del hioides y su localización respecto a las dimensiones anteroposteriores de la vía aérea superior, este se encuentra más anterior en pacientes clase III y en algunas ocasiones en angulación reversa. En comparación con los niños, las niñas con maloclusión clase III muestran una posición del hueso hioides más posterior, probablemente por la conciencia de su mandíbula prognática, lo que les hace asumir, desde muy temprano en la vida, una postura de la cabeza diferente para camuflar el mentón prominente [34, 37]. En las maloclusiones clase II, cuando el hioides está descendido, la lengua se posiciona más atrás reduciendo la permeabilidad de la vía aérea, lo cual se asocia con los pacientes que presentan apnea obstructiva del sueño y se reporta con más frecuencia en hombres [38].

El hueso hioides parece tener una posición constante en reposo, la cual no es afectada permanentemente por hábitos como el empuje lingual y la respiración oral [14], pero desciende progresivamente en tanto incrementa la resistencia en el flujo de aire en pacientes con deficiencias transversales maxilares [39].

Durante los tratamientos de ortodoncia, es posible disminuir el espacio detrás del velo del paladar y la lengua, cuando se retraen los dientes anteriores haciendo que el hioides se desplace en dirección posterior e inferior por adaptación para prevenir la invasión de la lengua en el espacio faríngeo [40]. Después de una cirugía de retroceso mandibular también ocurren estos cambios, buscando una protección de la vía aérea [41]. 


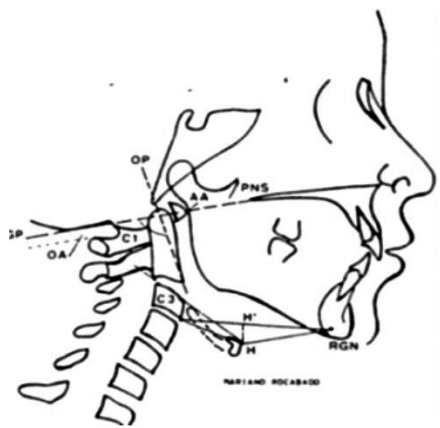

Figura 3. Puntos cefalométricos para evaluar el hueso hioides Fuente: Lin y Tsai , 2011 [40]

\section{Vías aéreas}

La actividad respiratoria normal tiene influencia en el crecimiento de las estructuras maxilofaciales, favoreciendo un crecimiento y un desarrollo adecuados. El conocimiento del crecimiento craneofacial, la morfología dentofacial y la función normal, es esencial para el diagnóstico, el plan de tratamiento y la estabilidad en la ortodoncia [42], mientras que un patrón respiratorio alterado puede cambiar la posición de la cabeza, la mandíbula y la lengua alterando el equilibrio de presiones y su posición [43].

Las radiografías cefálicas laterales se utilizan para evaluar las vías aéreas, aunque presentan limitaciones geométricas por ser una representación bidimensional del espacio faríngeo, y se requiere que el paciente tenga la lengua en posición de reposo durante la toma radiográfica, con el paladar blando descansando sobre el borde de la lengua [44].

Las dos medidas utilizadas son el espacio faríngeo superior $-\mathrm{o}$ nasofaringe $-\mathrm{y}$ el espacio faríngeo inferior -o la orofaringe. El espacio faríngeo superior es donde se localizan las adenoides y las amígdalas; es tomado desde el punto medio de la pared posterior del paladar blando hasta el punto más cercano sobre la pared faríngea posterior, y debe medir en promedio 17,4 $\mathrm{mm} \pm 4,3 \mathrm{~mm}$. El espacio faríngeo inferior -el cual se toma desde la intersección del borde posterior de la lengua y el borde inferior de la mandíbula hasta la pared posterior de la faringe- mide en promedio 11,3 $\mathrm{mm}$ en mujeres $\pm 3,3 \mathrm{~mm}$ y en hombres $13,5 \mathrm{~mm} \pm 4,3$ $\mathrm{mm}$ [42]. La amplitud de la vía aérea superior es similar entre géneros pero aumenta con la edad, mientras que la inferior es significativamente más amplia en mujeres que en hombres [42].
La respiración oral se vuelve necesaria cuando existe una obstrucción nasal $\mathrm{u}$ otros factores predisponentes, como amígdalas palatinas o faríngeas (adenoides) hipertróficas, o por enfermedades nasales (rinitis alérgica); asimismo por una anatomía de las vías aéreas estrechas y por factores ambientales irritantes predisponentes e infecciones [42, 45].

La apnea obstructiva del sueño - uno de los problemas más comunes de las vías aéreas- es una condición que potencialmente amenaza la vida, pues en ella el paciente sufre de ceses periódicos de la respiración durante el sueño por la reducción anteroposterior en las dimensiones de la vía aérea faríngea. La elongación y el engrosamiento del paladar blando, la dimensión grande de la lengua y la posición baja del hueso hioides en posición supina durante el sueño nocturno, obstruyen la vía aérea faríngea, lo cual afecta la calidad de vida. Los pacientes que presentan esta condición se caracterizan por presentar un retrognatismo tanto maxilar como mandibular, un incremento en la altura facial inferior y un ángulo cráneocervical amplio con una inclinación hacia delante de la columna cervical [44-46].

La posición de los dientes en un individuo está en equilibrio con los tejidos blandos orales (labios, lengua $y$ carrillos), y es afectada por varias funciones: respiración, deglución, masticación y fonación [42].

La influencia de la obstrucción de las vías respiratorias en el desarrollo del patrón de la cara es controversial. Muchos estudios en la literatura han tratado de explicar y vincular la obstrucción de las vías respiratorias y el crecimiento anormal craneofacial [44]. Algunos autores asocian la obstrucción nasal posterior con la rotación posterior de la mandíbula, crecimiento posterior-superior condilar, ángulo mandibular obtuso, mordida abierta anterior, arcos dentales estrechos, aumento de la altura inferior, retrognatismo mandibular, bóveda palatina profunda, incisivos proinclinados y mordida cruzada posterior, además de ausencia de selle labial y posición adelantada de la lengua y la cabeza para mantener la permeabilidad de las vías respiratorias $[42,43,45,47]$.

\section{Morfología de la sínfisis mandibular}

El conocimiento del crecimiento mandibular es altamente beneficioso en el diagnóstico y la planificación del tratamiento. Para predecir los patrones de crecimiento anterior y posterior se han utilizado varios 
parámetros cefalométricos que todavía son pobres en su precisión. Ricketts y otros investigadores indican que la morfología de la sínfisis mandibular puede ser usada para este fin [48].

Las dimensiones de la sínfisis continúan cambiando hasta la edad adulta y son los hombres quienes tienen una relación más fuerte entre la morfología de la sínfisis y la dirección del crecimiento mandibular, en comparación con las mujeres [48].

Una mandíbula con crecimiento anterior se asocia con una altura pequeña, profundidad grande, un radio pequeño y gran ángulo de la sínfisis. A la inversa, una dirección de crecimiento posterior se asocia con una gran altura, profundidad pequeña, radio grande y el ángulo pequeño de la sínfisis (figura 4) [48, 49].

La sínfisis mandibular es un factor anatómico que limita el movimiento de los incisivos y se debe evaluar para reducir el riesgo de daño radicular y al hueso alveolar. Los dientes con más riesgo están relacionados con las sínfisis más delgadas, ya que tiene menor hueso esponjoso [50].
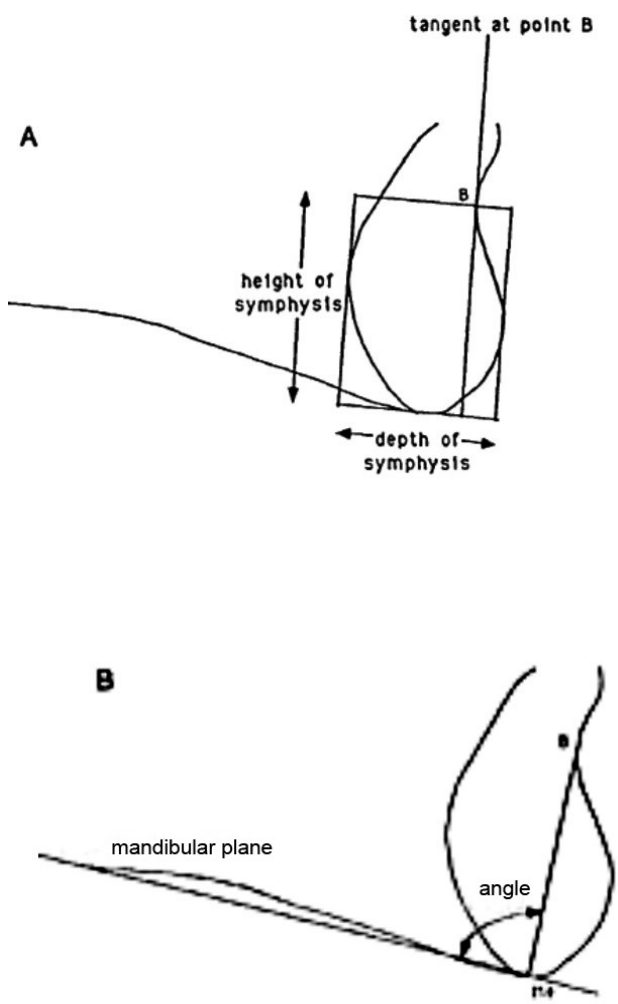

Figura 4. Mediciones cefalométricas utilizadas para cuantificar la morfología de la sínfisis

Fuente: Aki, 1994 [48]

\section{Conclusión}

La radiografía cefálica lateral da una visión más amplia de los patrones de crecimiento craneofacial, los cuales pueden verse modificados por diversas estructuras como la base del cráneo, la postura craneocervical, las vías áreas y posición del hueso hioides, sin dejar de valorar las vértebras cervicales y la morfología de la sínfisis mandibular, las cuales son factores diagnósticos importantes en el momento de tomar decisiones clínicas y evaluar la estabilidad.

La radiografía cefálica lateral como medio diagnóstico en ortodoncia, no sólo le proporciona al clínico información cuantitativa hallada en las medidas angulares y lineales de las estructuras craneofaciales, sino que, además, brinda información cualitativa importante que no se puede obviar y que permite llegar a un diagnóstico más acertado.

\section{Referencias}

[1] Solow B, Siersbaek-Nielsen S. Cervical and craniocervical posture as predictors of craniofacial growth. Am J Orthod Dentofacial Orthop. 1992; 101(5): 449-58.

[2] Wilhelm BM, Beck FM, Lidral AC, Vig KW. A comparison of cranial base growth in Class I and Class II skeletal patterns. Am J Orthod Dentofacial Orthop. 2001; 119(4): 401-5

[3] Henneberke M, Prahl-Andersen B. Cranial base growth for Dutch boys and girls: a multilevel approach. Am J Orthod Dentofacial Orthop. 1994; 106(5): 503-12.

[4] Dhopatkar A, Bhatia S, Rock P. An investigation into the relationship between the cranial base angle and malocclusion. Angle Orthod. 2002; 72(5): 456-63.

[5] Hopkin GB, Houston W, James GA. The cranial base as an aetiological factor in malocclusion. Angle Orthod. 1968; 38(3): 250-5.

[6] Ford MD. Growth of the human cranial base. Am J Orthodontics. 1958; 7(44): 498-506.

[7] Malta LA, Ortolani CF, Faltin K. Quantification of cranial base growth during pubertal growth. J Orthod. 2009; 36(4): 229-35.

[8] Lei WY, Wong RW, Rabie AB. Factors regulating endochondral ossification in the spheno-occipital synchondrosis. Angle Orthod. 2008; 78(2): 215-20.

[9] Björk A. Cranial base development. The Eleventh International Dental Congress Scientific Section. London July 19 to 261952.

[10] Latham RA. The sella point and postnatal growth of the human cranial base. Am J Orthod. 1972; 61(2): 156-62. 
[11] Ruiz AJ, Canseco J, Cuairán V. Relationship between cranial base flexure and skeletal class. Rev Odont Mex. 2011;.15(4): 214-8.

[12] Andria LM, Leite LP, Prevatte TM, King LB. Correlation of the cranial base angle and its components with other dental/skeletal variables and treatment time. Angle Orthod. 2004; 74(3): 361-6.

[13] Proff P, Will F, Bokan I, Fanghanel J, Gedrange T. Cranial base features in skeletal Class III patients. Angle Orthod. 2008; 78(3): 433-9.

[14] Klocke A, Nanda RS, Kahl-Nieke B. Role of cranial base flexure in developing sagittal jaw discrepancies. Am J Orthod Dentofacial Orthop. 2002; 122(4): 386-91.

[15] Anderson D, Popovich F. Correlations among craniofacial angles and dimensions in Class I and Class II malocclusions. Angle Orthod. 1989;.59(1):.37-42.

[16] Pachi F, Turla R, Checchi AP. Head posture and lower arch dental crowding. Angle Orthod. 2009; 79(5): 873-9.

[17] Garcia de Paula e Silva FW, Mussolino de Queiroz A, Díaz $\mathrm{KV}$. Posture alterations and consequence to stomatognatic system. Acta Odontol Venez. 2008; 46(4): 1-7.

[18] Solow B, Sandham A. Cranio-cervical posture: a factor in the development and function of the dentofacial structures. Eur J Orthod. 2002; 24(5): 447-56.

[19] Solow B, Siersbaek-Nielsen S. Growth changes in head posture related to craniofacial development. Am J Orthod. 1986; 89(2): 132-40.

[20] Cuccia AM, Lotti M, Caradonna D. Oral breathing and head posture. Angle Orthod. 2008; 78(1): 77-82.

[21] Huggare J. Association between morphology of the first cervical vertebra, head posture, and craniofacial structures. Eur J Orthod. 1991; 13: 435-40.

[22] Baydas B, Yavuz I, Durna N, Ceylan I. An investigation of cervicovertebral morphology in different sagittal skeletal growth patterns. Eur J Orthod. 2004; 26(1): 43-9.

[23] Gu Y, McNamara JA. Mandibular growth changes and cervical vertebral maturation. A cephalometric implant study. Angle Orthod. 2007; 77(6): 947-53.

[24] Franchi L, Baccetti T, Mcnamara J. Mandibular growth as related to cervical vertebral maturation and body heigth. Am J Orthod Dentofacial Orthop. 2000; 118: 335-40.

[25] Baccetti T, Franchi L, Mcnamara J. The cervical vertebral maturation (CVM) method for the assessment of optimal treatment timing in dentofacial orthopedics. Semin orthod. 2005; 11: 119-29.

[26] Baccetti T, Franchi L, Mcnamara J. An improved version of the cervical vertebral maturation (CVM) method for the assessment of mandibular growth. Angle Orthod. 2002; 72: 316-23.
[27] Gandini P, Mancini M, Andreani F. A comparison of hand-wrist bone and cervical vertebral analyses in measuring skeletal maturation. Angle Orthod. 2006; 76(6): 984-9.

[28] Lamparski D. Skeletal age assessment utilizing cervical vertebrae. [thesis]. Pittsburg: University of Pittsburg; 1972.

[29] Fudalej P, Bollen AM. Effectiveness of the cervical vertebral maturation method to predict postpeak circumpubertal growth of craniofacial structures. Am J Orthod Dentofacial Orthop. 2010; 137(1): 59-65.

[30] Soegiharto BM, Moles DR, Cunningham SJ. Discriminatory ability of the skeletal maturation index and the cervical vertebrae maturation index in detecting peak pubertal growth in Indonesian and white subjects with receiver operating characteristics analysis. Am J Orthod Dentofacial Orthop. 2008; 134(2): 227-37.

[31] Zhao XG, Lin J, Jiang JH, Wang Q, Hong S. Validity and reliability of a method for assessment of cervical vertebral maturation. Angle Orthod. 2012; 82(2): 229-34.

[32] Carulla D, Espinosa D, Mesa T. Estudio cefalométrico del hueso hioides en niños respiradores bucales de 11 años (I parte). Rev Cubana Estomatol. 2008; 45(2): versión On-line.

[33] Sheng CM, Lin LH, Su Y, Tsai HH. Developmental Changes in Pharyngeal Airway Depth and Hyoid Bone Position from Childhood to Young Adulthood. Angle Orthod. 2009; 79: 484-90.

[34] Adamidis IP, Spyropoulos MN. Hyoid bone position and orientation in class I and class III malocclusions. Am J Orthod Dentofacial Orthop. 1992; 101: 308-12.

[35] Pae EK, Quas C, Quas J, Garrett N. Can facial type be used to predict changes in hyoid bone position with age? A perspective based on longitudinal data. Am J Orthod Dentofacial Orthop. 2008; 134: 792-7.

[36] Jena AK, Duggal R. Hyoid bone position in subjects with different vertical jaw dysplasias. Angle Orthod. 2011; 81(1): 81-5.

[37] Yamaoka M, Furusawa K, Uematsu T, Okafuji N, Kayamoto D, Kurihara S. Relationship of the hyoid bone and posterior surface of the tongue in prognathism and micrognathia. J Oral Rehabil. 2003; 30(9): 914-20.

[38] Kollias I, Krogstad O. Adult craniocervical and pharyngeal changes. A longitudinal cephalometric study between 22 and 42 years of age. Part II: Morphological uvulo-glossopharyngeal changes. Eur J Orthod. 1999; 21(4): 345-55.

[39] Phoenix A, Valiathan M, Nelson S, Strohl KP, Hans M. Changes in hyoid bone position following rapid maxillary expansion in adolescents. Angle Orthod. 2011; 81(4): 632-8. 
[40] Lin YC LH, Tsai HH. Changes in the Pharyngeal Airway and Position of the Hyoid Bone After Treatment with a Modified Bionator in Growing Patients With Retrognathia. J Exp Clin Med. 2011; 3(2): 93-8.

[41] Graber LW. Hyoid changes following orthopedic treatment of mandibular prognathism. Angle Orthod. 1978; 48(1): 33-8.

[42] Goncalves R de C, Raveli DB, Pinto Ados S. Effects of age and gender on upper airway, lower airway and upper lip growth. Braz Oral Res. 2011; 25(3): 241-7.

[43] Martin O, Muelas L, Vinas MJ. Nasopharyngeal cephalometric study of ideal occlusions. Am J Orthod Dentofacial Orthop. 2006; 130(4): 436 e1-9.

[44] Solow B, Skov S, Ovesen J, Norup PW, Wildschiodtz G. Airway dimensions and head posture in obstructive sleep apnoea. Eur J Orthod. 1996; 18(6): 571-9.

[45] Gungor AY, Turkkahraman H. Effects of airway problems on maxillary growth: a review. Eur J Dent. 2009; 3(3): 250-4.
[46] Ingman T, Nieminen T, Hurmerinta K. Cephalometric comparison of pharyngeal changes in subjects with upper airway resistance syndrome or obstructive sleep apnoea in upright and supine positions. Eur J Orthod. 2004; 26(3): 321-6.

[47] Behlfelt K, Linder-Aronson S, McWilliam J, Neander P, Laage-Hellman J. Dentition in children with enlarged tonsils compared to control children. Eur J Orthod. 1989; 11(4): 416-29.

[48] Aki T, Nanda RS, Currier GF, Nanda SK. Assessment of symphysis morphology as a predictor of the direction of mandibular growth. Am J Orthod Dentofacial Orthop. 1994; 106(1): 60-9.

[49] Mangla R, Singh N, Dua V, Padmanabhan P, Khanna M. Evaluation of mandibular morphology in different facial types. Contemp Clin Dent. 2011; 2(3): 200-6.

[50] Gracco A, Luca L, Bongiorno MC, Siciliani G. Computed tomography evaluation of mandibular incisor bony support in untreated patients. Am J Orthod Dentofacial Orthop. 2010; 138(2): 179-87. 
